\title{
An update on long intergenic noncoding RNA p21: a regulatory molecule with various significant functions in cancer
}

\author{
Roya Amirinejad', Mina Rezaei ${ }^{2}$ and Zeinab Shirvani-Farsani ${ }^{2 *}$ (1)
}

\begin{abstract}
Long intergenic noncoding RNA p21 was mapped on the human chromosome 6p21.2. Accordingly, it was firstly described by promoting the p53-dependent apoptosis in the mouse. Also, it is a new IncRNA playing some vital roles in the cell cycle, apoptosis, cell proliferation, tumorigenesis, invasion, metastasis, and angiogenesis. In this regard, it was shown that, lincRNA-p21 regulates these biological processes involved in carcinogenesis through various signaling pathways including Notch signaling, JAK2/STAT3, and AKT/mTOR pathways. Another mechanism by that lincRNAp21 can affect these processes is a cross-talk with different miRNAs. In vitro and in vivo studies revealed dysregulation of lincRNA-p21 in various human cancers. In addition, emerging evidence demonstrated that, lincRNA-p21 can be considered as a potential prognostic and therapeutic biomarker in cancers. Also, lincRNA-p21 enhances the response to radiotherapy for colorectal cancer. However, the molecular mechanisms of lincRNA-p21 in carcinogenesis have not been fully elucidated so far. So, this review summarizes the function of lincRNA-p21, as a tumor suppressor factor in different biological processes implicated in cancers.
\end{abstract}

Keywords: lincRNA-p21, Biomarker, miRNAs, Metastasis, Angiogenesis, Apoptosis

\section{Background}

LincRNA-p21 (Long intergenic noncoding RNA p21,), which was mapped on human the chromosome $6 \mathrm{p} 21.2$, upstream of the cell-cycle regulator gene p21/Cdkn1a, was firstly described as an inducer of the p53-dependent apoptosis in mouse embryonic fibroblasts [1]. Lin$c R N A-p 21$ has two isoforms that both of them contain a single exon and Alu inverted repeat elements. Also, LincRNA- $p 21$ together with lncRNA NEAT1 localizes in HCT-116, MCF-7, and U2OS cells [2]. LincRNA-p21, also called as TRP53COR1 (P53 Pathway Corepressor 1 protein tumor), is a new lncRNA that has an important function in initiating and progressing various cancers.

*Correspondence: z_shirvani@sbu.ac.ir

2 Department of Cell and Molecular Biology, Faculty of Life Sciences and Biotechnology, Shahid Beheshti University G.C, Tehran, IR, Iran

Full list of author information is available at the end of the article
Moreover, it was shown that, $\operatorname{lin} c R N A-p 21$ induces the transcriptional activity of wild-type p53 on vascular smooth muscle cells [3]. Also, it suppresses expression through either targeting mRNAs or recruiting protein binding partners to the chromatin location [4], because it contains several motifs interacting with the mRNA targets, different miRNAs, and RNA-binding proteins [5]. In addition, multiple evidence has reported that, lincRNA-p21 involves in the development of different tumors. Accordingly, the lincRNA-p21 overexpression can suppress tumor invasion by Notch pathway [6]. Also, lincRNA-p21 suppresses the progression of prostate cancer by apoptotic induction and controlling gene expressions downstream of p53 [7]. In fact, it plays a significant role as a moderator in different processes such as DNA damage response, apoptosis, and in cell proliferation [8]. However, the mechanisms and functions of lincRNA-p21 are still unknown in the progression and development 
of various cancers. Likewise, lincRNA-p21 are induced by DNA damage response. Then, lincRNA- $p 21$ recruit hnRNP-K (heterogeneous nuclear ribonucleoprotein $\mathrm{K}$ ) on the promoter of $\mathrm{p} 21$, which is crucial for the interaction between the p53 and the $\mathrm{p} 21$ promoters and eventually in the enhancement of p21 expression [4]. In vivo and in vitro studies of cancer have indicated the tumor suppressor functions of lincRNA-p21 in different cancer types [9-11]. Several studies have found that, lincRNA$p 21$ has significantly reduced in multiple cancers such as gastric cancer [12], chronic lymphocytic leukemia [13], hepatocellular carcinoma [6], non-small cell lung cancer [14], colorectal cancer [15], diffuse large B cell lymphoma [16], and prostate cancer [7]. Besides presenting regulatory function, lincRNA-p21 was detectable in body fluids such as blood, serum, plasma, and urine. Therefore, it can be suggested as a potential biomarker for prostate cancer [17]. Overall, few studies have been conducted on various cancers, the results of which are summarized in Table 1 . In this review, we discussed the functions of lincRNAp21 in various biological processes including tumorigenesis, and then described further evidence for the effects of lincRNA-p21 on the signaling pathways as well as a cross-talk between lincRNA-p21 and miRNAs involved in cancers.

\section{LincRNA-p21 in apoptosis, cell proliferation, and cell cycle}

Recent studies have indicated that, lincRNA-p21 is a significant regulator of apoptosis and cell proliferation by p53 signaling and inhibiting the target gene translation. For instance, Huarte et al. [1] found that, lincRNA-p21 with p53 can control the number of apoptosis and cell proliferation regulator genes. They also observed that, lincRNA-p 21 and $\mathrm{p} 53$ knockdowns lead to the inhibition of the apoptosis genes such as Perp and Noxa, as well as the activation of cell survival genes such as Stat3, Atf2, and Bcl2l3. Also, lincRNA-p21 overexpression results in cellular apoptosis after the induction of DNA damage [1]. Moreover, Wu et al. [3] have reported that, lincRNA-p21 interacts with MDM2, thus releasing p53 from MDM2, and p53 binds to p300, resulting in the increase of p53 activity [3]. Moreover, another study demonstrated that, the apoptosis induction by ING1b is dependent on the lincRNA-p21 expression [18]. Accordingly, this impact has significantly increased by p53. Furthermore, ING1b can bind to the lincRNA-p21 promoter and also controls

Table 1 The pattern of lincRNA-p21 expression and its roles in various cancers

\begin{tabular}{|c|c|c|c|c|c|c|}
\hline \multirow[t]{2}{*}{ Cancer type } & \multicolumn{2}{|l|}{ Sample type } & \multirow[t]{2}{*}{ Expression pattern } & \multirow{2}{*}{$\begin{array}{l}\text { Gene interplay } \\
\text { (Direct or Indirect) }\end{array}$} & \multirow[t]{2}{*}{ Signaling pathways } & \multirow[t]{2}{*}{ Reference } \\
\hline & Cell line & Patients & & & & \\
\hline $\begin{array}{l}\text { Hepatocellular carci- } \\
\text { noma }\end{array}$ & $\begin{array}{l}\text { HepG2 and SMMC- } \\
7721\end{array}$ & HCC tumor tissue & Down-regulated & $\begin{array}{l}\text { Hes-1, NICD, } \\
\text { E-cadherin, and } \\
\text { Claudin-1 }\end{array}$ & $\begin{array}{l}\text { Notch and EMT } \\
\text { signal pathways }\end{array}$ & {$[6]$} \\
\hline Prostate cancer & $\begin{array}{l}\text { LNCaP, DU145, PC3, } \\
\text { PTN2 and BPH-1 }\end{array}$ & $\begin{array}{l}\text { Prostatectomy } \\
\text { specimens }\end{array}$ & Down-regulated & $\begin{array}{l}\text { p53, Mdm2, Puma, } \\
\text { Noxa and Bax }\end{array}$ & P53 Signaling & {$[7]$} \\
\hline $\begin{array}{l}\text { Head and neck } \\
\text { squamous cell } \\
\text { carcinoma }\end{array}$ & $\begin{array}{l}\text { HN4, HN6, HN30 } \\
\text { HEK293T, Cal27, } \\
\text { SCC25, Detroit562, } \\
\text { MCF7, and MDA- } \\
\text { MB-231 }\end{array}$ & HNSCC tumor tissues & Down-regulated & p53 and NF-YA & JAK2/STAT3 signaling & {$[9]$} \\
\hline Skin cancer & $\begin{array}{l}\text { Balb/MK2 keratino- } \\
\text { cytes, NHEKs }\end{array}$ & - & Down-regulated & P53 & p53 pathway & {$[10]$} \\
\hline Gastric cancer & $\begin{array}{l}\text { MGC-803, MKN-45, } \\
\text { BGC-823, MKN-28, } \\
\text { SGC-7901 and } \\
\text { normal GES-1 }\end{array}$ & GC tissues & Down-regulated & $\begin{array}{l}\text { imentin and } \\
\text { N-cadherin, YAP, } \\
\beta \text {-catenin and } \\
\text { NF-KB, P-ERK }\end{array}$ & Hippo pathway & {$[12]$} \\
\hline $\begin{array}{l}\text { Chronic lymphocytic } \\
\text { leukemia }\end{array}$ & $\begin{array}{l}\mathrm{LY}-47, \mathrm{BL}-60 \text { and } B L-7 \text {, } \\
\text { BJAB, BL-2, and } \\
\text { CA-46 }\end{array}$ & PBMC from patients & $\begin{array}{l}\text { Up-regulated after } \\
\text { DNA damage }\end{array}$ & P53, p21 & p53 pathway & [13] \\
\hline $\begin{array}{l}\text { Non-small cell lung } \\
\text { cancer }\end{array}$ & $\begin{array}{l}\mathrm{H} 23, \mathrm{H} 1299 \text {, and } \\
\text { HCC-44 }\end{array}$ & NSCL tumor tissue & Down-regulated & $\begin{array}{l}\text { Secreted vascular } \\
\text { endothelial growth } \\
\text { factor A (VEGFA) }\end{array}$ & - & {$[14]$} \\
\hline Colorectal cancer & $\begin{array}{l}\text { CRC cell lines HCT- } \\
116(\mathrm{p} 53 \mathrm{p} / \mathrm{p}) \text { and } \\
\text { HCT-116 (p53-/-) }\end{array}$ & CRC specimens & Down-regulated & P53 & - & {$[15]$} \\
\hline B cell lymphoma & - & DLBCL tissues & Down-regulated & $\begin{array}{l}\text { Cyclin D1, CDK4 and } \\
\text { p21 }\end{array}$ & - & {$[16]$} \\
\hline
\end{tabular}


the level of lincRNA-p21. In addition, it is necessary for inducing lincRNA- 21 by p53 [18]. Therefore, lincRNA$p 21$ may cross-talk to $\mathrm{p} 53$ and ING1b signaling pathways (Fig. 1). Hall et al. [10] have also reported that, the downregulation of lincRNA-p21 suppressed UVB-induced apoptosis in keratinocytes from the human and mouse, while this reduced lincRNA-p21 expression had no impact on the cell proliferation in keratinocytes before and after the treatment with UVB. Also, a mutation in a single p53 allele blocks the activation of UVB-induced expression of lincRNA-p21, and then inhibits the apoptosis. Accordingly, this allele may be a pro-oncogenic allele in skin cancer [10]. On the contrary, Yang et al. [19] have observed that, lincRNA- $p 21$ was considerably enhanced in the tissues of human non-small cell lung cancer and cells thereby significantly suppressed the cell apoptosis. In other words, the overexpression of $\operatorname{lincRNA-p21}$ repressed apoptosis, while its knockdown increased the apoptosis. The impact of lincRNA-p21 on apoptosis was mediated by downregulation of the PUMA (p53 upregulated modulator of apoptosis) [19]. PUMA is a target of p53 by having significant functions in the regulation of apoptosis [20]. In fact, PUMA is a pro-apoptotic factor that represses some anti-apoptotic factors including Mcl-1, Bcl-2, Bcl-XL, Bcl-W, and A1 and also activates apoptosis [21].

The results of another investigation have demonstrated that, down-regulation of $l i n c R N A-p 21$ enhanced the percentage of the cells in $\mathrm{S}$ phase and decreased the apoptosis. Whereas, up-regulation of lincRNA-p21 resulted in G1 arrest in HN6 and Cal27 cells as well as reducing the expression levels of several cell cycle regulating factors such as Cyclin B1 and Cyclin D1 along with the apoptosis induction in head and neck squamous cell carcinoma (HNSCC) cells. Collectively, lincRNA-p21 suppressed the HNSCC cell growth, activated cell apoptosis, and induced G1 phase arrest in vitro and in vivo studies [9]. Likewise, lincRNA-p21 can diminish the expression of HIF- $1 \alpha$, reduce the VEGF levels, prevent the cell proliferation and invasion, and finally increase the apoptosis of $\mathrm{MHCC} 97 \mathrm{H}$

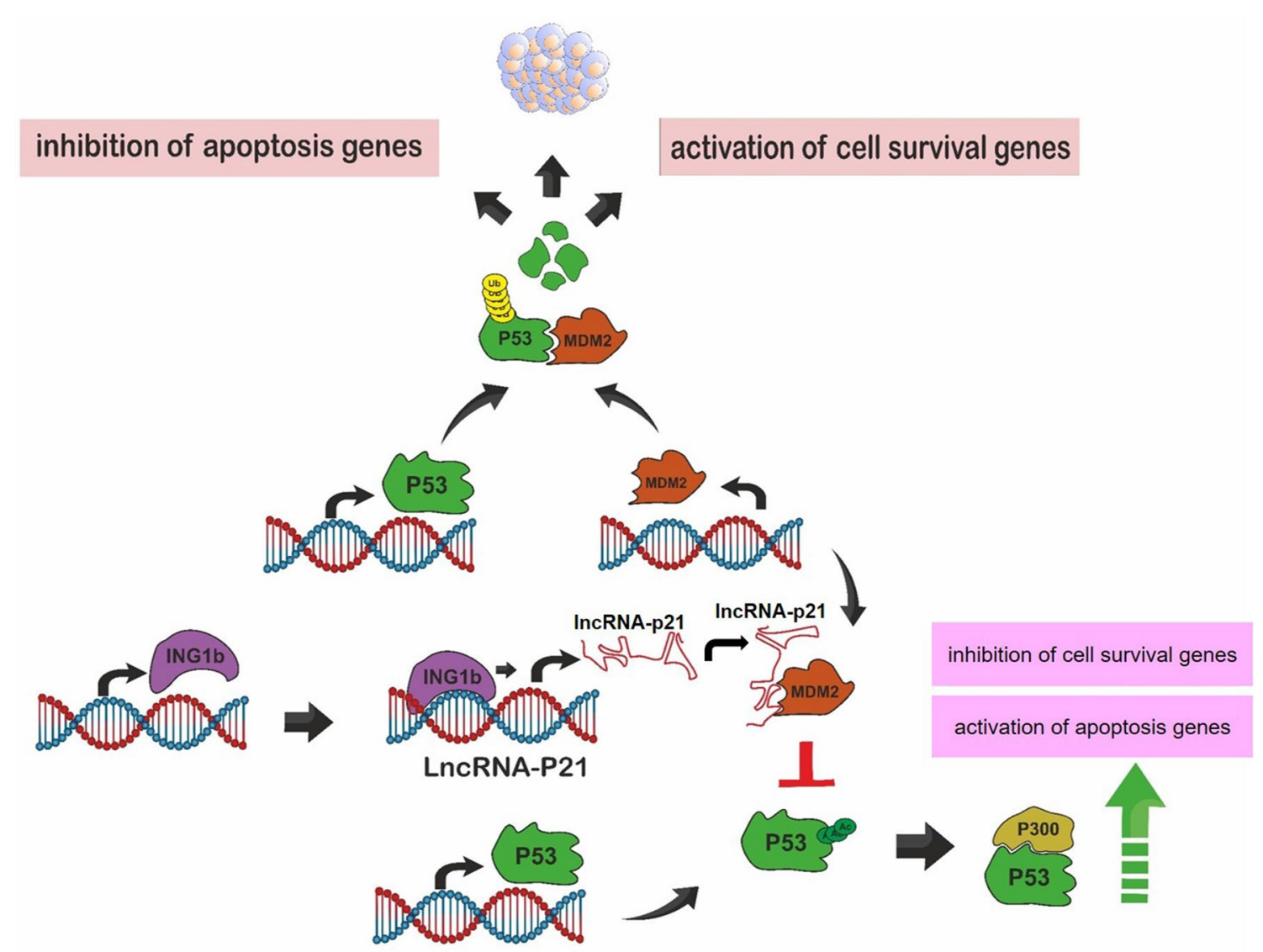

Fig. 1 LincRNA-p21-induced apoptosis via ING1B and p53. MDM2 monoubiquitinates p53, which leads to its degradation by the proteasome complex. Consequently, the apoptosis genes are inhibited and cell survival genes are activated, resulting in the inhibition of apoptosis (up). On the other hand, ING $1 \mathrm{~b}$ leads to the overexpression of lincRNA-p21. Then, lincRNA-p21 binds to MDM2 and detaches it from p53, which results in the increased p53 stability, the induced apoptosis genes, and the suppressed survival cell genes (down) 
liver cancer cells [22]. Similarly, the increased lincRNA$p 21$ expression suppresses invasion, the transition of the cell cycle from G1, cell proliferation, and migration, and also activates the apoptosis of the oesophageal cancer cell line. Actually, it seems that, lincRNA-p21 acts through enhancing the expression of $\mathrm{p} 21$ and reduces the expression of cyclin D, and as a result, cell-cycle were arrested [23]. In this context, silencing lincRNA-p21 lead to the reduced levels of $\mathrm{p} 21$, rearrangement of chromatin state of some target genes of polycomb, diminish the G1/S efficiency, and enhance the cell proliferation [4]. Another study indicated the enhanced lincRNA-p21 levels and the decreased cell proliferation in mesenchymal stem cells isolated from aged mice compared to younger mice. Notably, the lincRNA- $p 21$ knockdown increased the cell growth via the $W n t / \beta$-catenin signaling pathway [24]. Although lincRNA-p21 may be considered as a tumour suppressor that induce apoptosis, prevent cell growth, and arrest cycle progression, understanding the precise mechanisms of lincRNA-p21 in different processes in carcinogenesis needs further study.

\section{LincRNA-p21 in tumorigenesis}

Accumulated evidence has been demonstrated that, lncRNAs including lincRNA-p21 play critical roles in tumorigenesis. In fact, lincRNA-p21 inhibits $\beta$-catenin translation, leads to the down-regulation of protein levels of $\beta$-catenin in HeLa cells [25], which suggest that, it has a potential suppressor of tumorigenesis. However, lin$c R N A-p 21$ enhances HIF- $1 \alpha$ stability under the hypoxia conditions points that it may also have an oncogenic function and facilitate tumorigenesis [11, 26]. LincRNA$p 21$ directly binds to STAT3 resulting in the prevention of tumorigenic signals in HNSCC. Briefly, the downregulation of lincRNA-p21 reduces its interaction with STAT3, and consequently activates the transcriptional activity of STAT3 and promotes tumor progression. On the other hand, lincRNA-p21 up-regulation suppresses the regulatory activity of STAT3, as a result, it inhibits tumorigenesis [9] (Fig. 2). In addition, the low expression of lincRNA-p21 in cancer stem cells led to the activation of tumorigenesis, whereas its increased expression potently repressed the tumorigenesis [27]. Also, Zhang et al. [23] indicated that, lincRNA-p21 inhibits tumorigenesis in esophageal squamous cell carcinoma. Meanwhile, knockdown studies indicated that, endogenous lincRNA-p21 inhibited tumorigenesis and differentiation [28]. Therefore, the lincRNA-p21 has been suggested, as a potent suppressor of tumorigenesis, which could be considered as a valuable therapeutic biomarker in various cancers. For example, the expression of lincRNA-p21 by a novel adenoviral vector in cancer stem cells, could inhibit $\mathrm{Wnt} / \beta$-catenin signaling pathway resulted in repressing

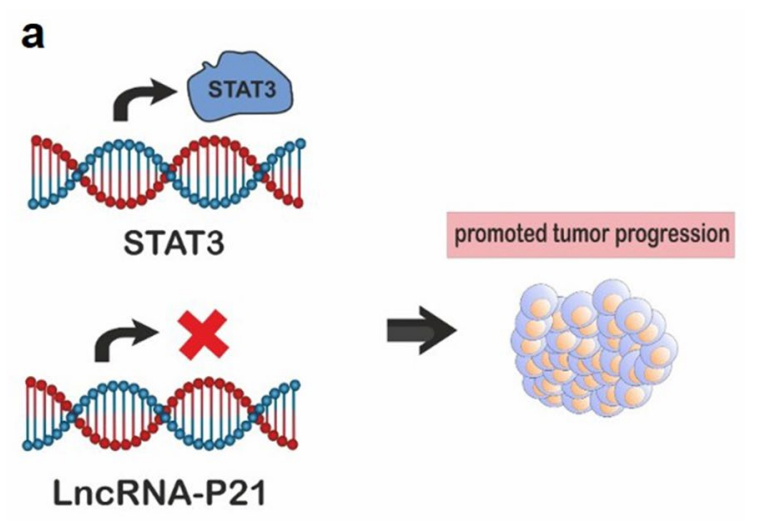

b
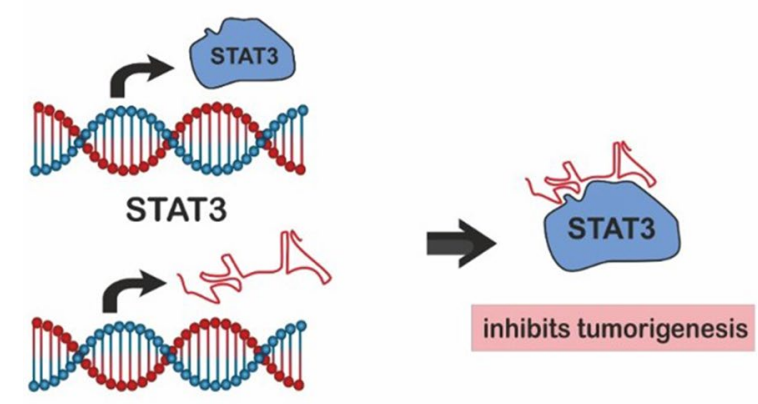

LncRNA-P21

Fig. 2 Cross-talk between lincRNA-p21 and STAT3. a The reduced expression of lincRNA-p21 inhibits its interaction to STAT3, thus promotes the STAT3 transcriptional activity and induces tumor progression. $\mathbf{b}$ Overexpression of lincRNA-p21 represses the regulatory activity STAT3 and also inhibits tumorigenesis

the viability and tumorgenesis of these cells [27]. In addition, Isin et al., reported that, the expression levels of lin$c R N A-p 21$ could be a potential diagnostic biomarker in the patients with prostate cancer [29].

\section{LincRNA-p21 in cancer invasion and metastasis}

Tumor invasion and metastasis, which are the symptoms of disease development and the main causes of treatment failure, are known as the main reasons for mortality. Although a number of investigations have assessed the tumor invasion and metastasis, its exact mechanism is not known yet [30]. Previous studies have indicated that, genetic factors including small non-coding RNA and lncRNAs play important roles in the progression of various cancers and malignant metastasis [31, 32].

A number of studies have reported that, lncRNAs including lincRNA-p21 can inhibit the invasion and metastasis of different cancers such as gastric cancer, hepatocellular carcinoma, and colorectal cancer. For 
example, Chen et al. [12] reported that, in gastric cancer cell lines, cell-to-cell contact was lost and also gained a spindle-like appearance, and vimentin and $\mathrm{N}$-cadherin have enhanced after a lincRNA-p21 knockdown by the Hippo pathway and YAP activation [12].

Moreover, it has been revealed that, IincRNA-p21 activates the epithelial-mesenchymal transition (EMT) by downstream miRNA [33], while Jia et al. [6] have reported that, the increased lincRNA-p21 expression can suppress EMT via Notch singling, along with the reduced lincRNA-p21 expression that led to a reverse result [6]. In this regard, lncRNA-p21 has been indicated to control the of microRNA-9 expression level negatively. Moreover, microRNA-9 negatively regulates E-cadherin and cell adhesion. Therefore, lincRNA-p21 could repress the development of hepatocellular carcinoma by the miR9/E-cadherin signaling pathway [33]. Furthermore, high lincRNA-p21 expression reduced the migration and invasion abilities of the head and neck squamous cell carcinoma (HNSCC) cell lines (HN6 and Cal27 cells); however, they have enhanced after the down-regulation of lincRNA-p21. Correspondingly, the up-regulation of lincRNA- $p 21$ resulted in the decreased matrix metalloproteinase 2 (MMP2) and MMP9 proteins, and vice versa. These resulted proved that, lincRNA-p21 expression suppressed the aggressive manners in these cells [9] (Fig. 3).

\section{LincRNA-p21 in angiogenesis}

Angiogenesis and lymph angiogenesis promoted by chemical factors from tumor cells, are key factors in the growth of tumor and metastasis. Vasculogenesis leads to the formation of the initial vascular network, and consequently differentiate endothelial cells (ECs) from precursors including angioblast. Afterward, angiogenesis activates the formation of the new blood vessels from the existing vessels. In these processes, angiogenic factors such as vascular endothelial growth factor (VEGF) or hypoxia play the key roles $[34,35]$.

Previous studies have reported that, there are different signaling pathways and new molecules for the induction of angiogenesis. Also, one of the most important novel markers in angiogenesis is lncRNAs [36]. LincRNA-p 21 has recently been reported to have critical functions in angiogenesis. For example, in a very interesting study, Castellano et al. [14] found that, lin$c R N A-p 21$ play a prognostic role in NSCLC (Non-Small Cell Lung Cancer) via controlling angiogenesis. In this regard, they observed that, the downregulation of lin$c R N A-p 21$ under hypoxic conditions causes a reduction in the expression of genes related to angiogenesis (MMP2, PDGFB, VEGFA, FGF2, granulin, and EDIL3). These genes are involved in various processes such as extracellular matrix degradation, metastasis, formation in endothelial cells, angiogenesis, and cell motility. In

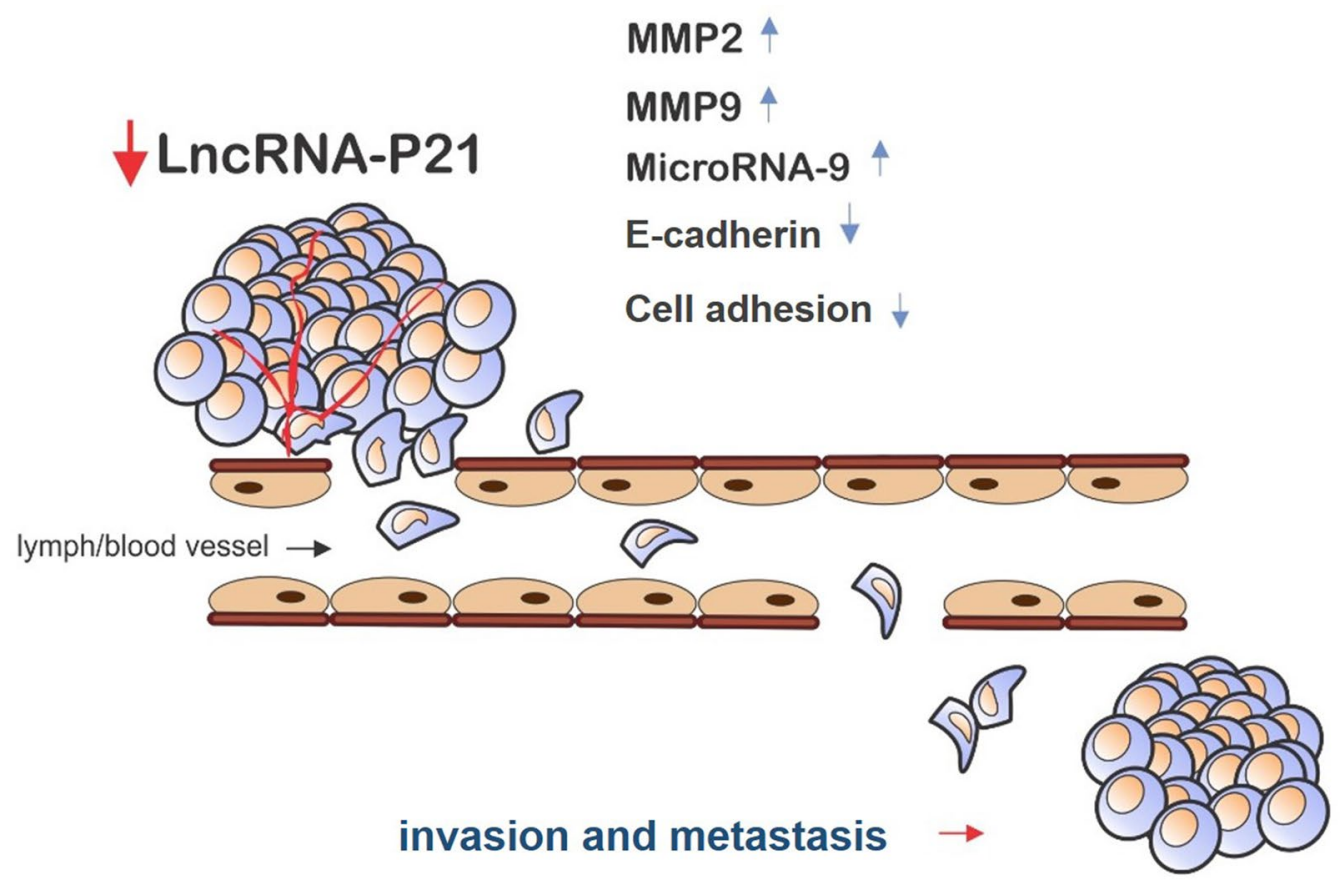

Fig. 3 The role of lincRNA-p21 in invasion and metastasis. Down-regulation of lincRNA-p21 leads to enhance MMP2, MMP9, and miRNA-9 expression, as well as reducing the E-cadherin expression and cell adhesion, which finally promotes invasion and metastasis 
addition, after silencing lincRNA-p21, HUVEC tube formation reduced, which suggest a regulatory function of lincRNA-p21 in angiogenesis. Interestingly, the increased levels of lincRNA-p21 in tumor cells from the patients with NSCLC were related to the higher microvascular density [14]. While the inhibition of lincRNA$p 21$ expression activated angiogenesis in the rats with rheumatoid arthritis (RA). In addition, there was no significant difference in the levels of bFGF, VEGF, and HGF expressions between the RA rats and the RA rats with down-regulating lincRNA-p21 [37]. Also, determining the precise role of lincRNA-p21 in the angiogenesis by in vivo models may provide a promising therapeutic marker.

\section{LincRNA-P21 in different signaling pathways}

Several studies have evaluated the expression levels of lincRNA-p21 in the different cancer's tissues and cell lines. Furthermore, numerous studies have revealed the role of this lncRNA in suppressing the cell proliferation and cell growth, as well as the enhancement of apoptosis. Also, it is worth mentioning that, lincRNA-p21 is able to regulate some important signaling pathways in cell proliferation, cell growth, cell cycle, apoptosis, and metastasis including Wnt/ $\beta$-catenin, miR-9/E-cadherin, Notch signaling, JAK2/STAT3, and AKT/mTOR pathways $[6,9,33,38,39]$. In this regard, Wang et al. [39] found that, knockdown of linc-RNA- $p 21$ leads to the increased PKM2 expression as well as the activation of AKT or mTOR signaling. PI3K/AKT/mTOR signaling pathway has an important function in cancer progression, such as the prostate cancer [40]. Similarly, the high expression of lincRNA-p21 results in the inhibition of the Wnt/ $\beta$ catenin pathway, and consequently inactivate the hepatic stellate cells (HSCs) via the impact of miR-17-5p on Wnt inhibitory factor 1 (WIF1) [38] (Fig. 4). In this regard, lin$c R N A-p 21$ may enhance the response to radiotherapy for colorectal cancer through the inhibition of the $\beta$-catenin signaling pathway and increase in the expression of the Noxa, as a pro-apoptosis gene [41]. Interestingly, the upregulation of lincRNA- $p 21$ inhibited cell proliferation and the expression of $\alpha$-SMA and type I collagen in the hepatic stellate cells (HSC). Actually, lincRNA-p21 acts through microRNA-181b to enhance the expressions of PTEN and HSC activation (lincRNA-p21-miR-181bPTEN Signaling pathway) [42]. Similarly, lincRNA-p21 suppressed the progression of lung cancer cells via the inhibition of cell proliferation and migration as well as the activation of apoptosis in NSCLC. Accordingly, this impact of lincRNA-p21 on lung cancer cells is mediated by the miR-17-5p signaling pathway. Notably, miR-17-5p is a target of lincRNA-p21 [43].

\section{Crosstalk between lincRNA-p21 and miRNAs}

lncRNAs have been indicated to have an interaction with other RNAs including miRNAs via nucleic-acid base pairing, and this interaction leads to the lncRNA-mRNA competition for microRNA binding. Based on this fact, lncRNAs are believed to be new competing endogenous RNAs (ceRNAs), which present miRNA binding sites (MREs) $[44,45]$. In order for a lncRNA to act as a ceRNA, an MRE in lncRNA requires an incomplete complement to bind to the miRNA. Therefore, the interaction of lncRNAs with miRNAs causes a slow degradation [46]. Novel growing evidence demonstrates that, the interaction between lncRNAs as ceRNAs and miRNAs can regulate various cellular processes and genetic pathways. Also, the disruption of the ceRNAs functions can affect the diverse processes and human diseases including cancer [47]. For instance, Ye et al. [47] by in silico prediction showed that, lincRNA-p21 has a putative MRE for the miR-181 family. They observed that, lincRNA-p21 binds to the miR-181 family and also forms a negative feedback loop with miR$181 / \mathrm{PKC}-\delta$ that promotes the microglial activation [47]. In line with these results, it was shown that, lincRNA$p 21$ could inhibit $\mathrm{Wnt} / \beta$-catenin pathway via binding to miR-17-5p in hepatic stellate cells [38]. Also, an in vitro study displayed the ability of lincRNA-p21 for binding to miR-1277-5p and regulation of the level of miR1277-5p expression. In addition, the up-regulation of miR-1277-5p inhibited the impact of lincRNA-p21 on the apoptosis and cell viability [48]. Interestingly, miR-320 family ( $\mathrm{a}, \mathrm{b}$ and $\mathrm{c}$ ) was reported to bind to a $5^{\prime}$ sequence of lincRNA- 21 and regulate its expression through HSF1 (heat-shock factor 1) in MDA-MB-231 cells [49] (Fig. 5).

Mechanisms of the cross-regulation between lncRNAs and miRNAs consisted of four posttranscriptional mechanisms. At first, miRNAs reduced the lncRNAs stabilities, which resulted in the modulation of lncRNAs amount abundance and affected different cellular processes [50]. For example, binding HuR, an RNAbinding protein, to lincRNA-p21 led to the recruitment of let-7/Ago2 to lincRNA-p21, and the decreased stability of lincRNA-p21. In fact, overexpression of let-7b activated the RNA degradation coding lincRNA-p21 in human cervical carcinoma HeLa cells [25]. Secondly, LncRNAs could sequester miRNAs away from their target mRNAs. Accordingly, these lncRNAs are known as 'sponges' or 'decoys' for miRNAs, which reduce available miRNAs abundance and increase translations of mRNAs [50]. Interestingly, it has been found that, lncRNA-p21 sponges miR 451 to activate apoptosis in osteoarthritis. In fact, the upregulation of lncRNAp21 inhibited the miR-451 expression [51]. An in vitro study reported that, LncRNA-p21 elevated the progression of diabetic nephropathy by acting as a sponge 
a

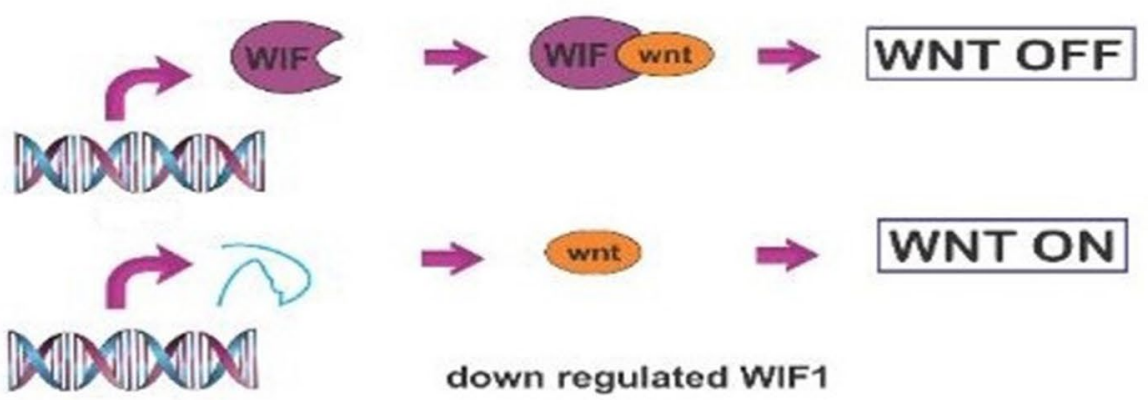

down regulated WIF1

b

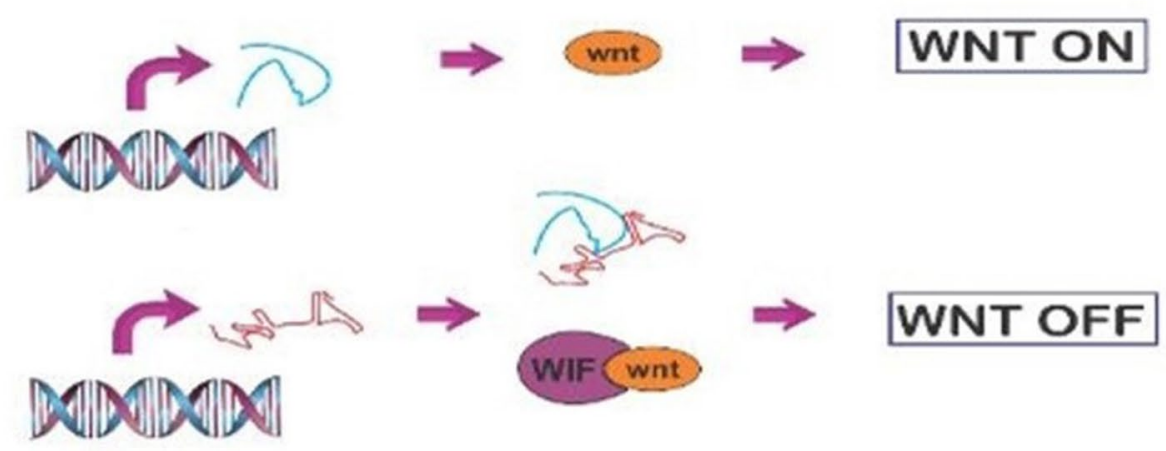

WNT ON

C

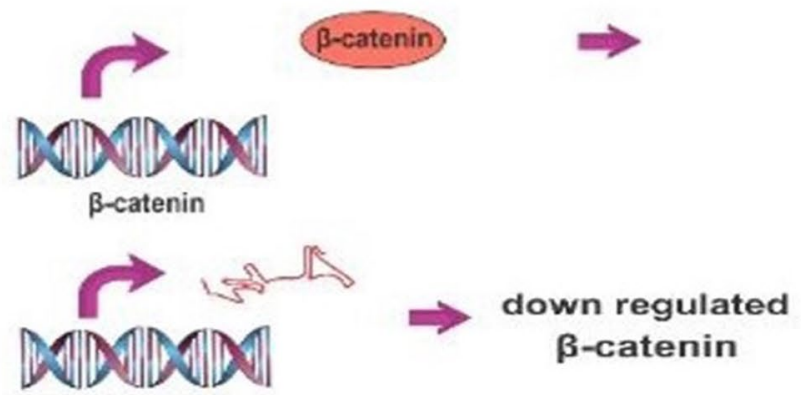

WNT ON

Fig. 4 LincRNA-p21 inhibits Wnt/B-catenin pathway. a miR-17-5p activates Wnt through down-regulating WIF1. b LincRNA-p21 suppresses miR-17-5p leading to the inactivation of Wnt. c LincRNA-p21 down-regulates the $\beta$-catenin and inhibits Wnt. WIF1: Wnt inhibitory factor 1

for miR-18b. In this study, they found that, miR-18b has reduced by lincRNA-p21 [52]. Thirdly, LncRNAs could compete with miRNAs for binding to their target mRNAs, which consequently removed the regulatory influences of miRNA on mRNAs [50]. Forth, some
IncRNAs could generate miRNAs from intronic and exonic region [53]. Altogether, lincRNA-p21 can not only bind to miRNAs directly, as miRNA sponges, and modulate their roles, but it can also be targeted by different miRNAs regulating its expression and function. 


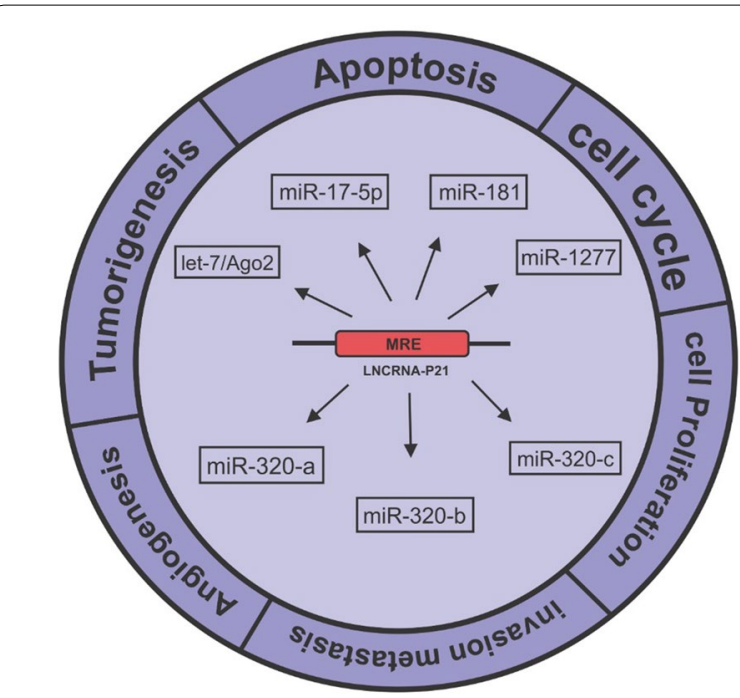

Fig. 5 The interplay between lincRNA-p21 and miRNAs for the regulation of various biological processes involved in cancer

\section{Conclusion and future perspectives}

Previous studies support the critical role of lincRNA$p 21$ in different processes occurring in human cancers. In fact, lincRNA-p21 acts as a tumour suppressor gene inhibiting the cell proliferation, cell survival, tumorigenesis, invasion, metastasis, and angiogenesis. On the other hand, it activates cell apoptosis, in which the expression of several genes happened. It seemed that, lincRNA-p21 may serve as one of the main regulators in various signaling pathways involved in carcinogenesis. It can also involve in response to different therapies for various cancers through regulating the signaling pathways. Moreover, lincRNA-p21 can affect different biological processes by interacting with miRNAs such as miR-181, miR-1277-5p, let-7, and miR-320. Notably, a combination of lincRNA-p21 along with several miRNAs can form a panel of potential biomarkers for various cancers. By indicating several vital roles of lnicRNAp 21 in the pathogenic processes involved in carcinogenesis and its dysregulated expression in human cancers, lincRNA-p21 is suggested as it may serve as a potential diagnostic and prognostic biomarker and a therapeutic target in human cancers. However, these biomarkers, especially in body fluids as a non-invasive way, must be validated using further clinical translation assessments. Meanwhile, the role of this lncRNA in response to radiotherapy suggests that, the regulation of this lincRNA may be a new therapeutic strategy for cancers. However, further clinical studies should be evaluated in the future. In addition, further research conducted on the functions of lincRNA-p21 can reveal the molecular mechanisms of cancer pathogenesis.

\section{Acknowledgements}

This study was technically supported by Shahid Beheshti Universty, Tehran, Iran.

\section{Authors' contributions}

RA and ZSh wrote and revised the manuscript. MR drew all Figures. All authors read and approved the final manuscript.

\section{Funding}

This research did not receive any specific grant from funding agencies in the public, commercial, or not-for-profit sectors.

\section{Availability of data and materials \\ Not applicable.}

\section{Ethics approval and consent to participate}

Not applicable.

\section{Consent for publication}

All authors agree to publish this paper.

\section{Competing interests}

The authors declare that they have no competing interests.

\section{Author details}

${ }^{1}$ Genetics Department, Breast Cancer Research Center, Motamed Center Institute, ACECR, Tehran, IR, Iran. ${ }^{2}$ Department of Cell and Molecular Biology, Faculty of Life Sciences and Biotechnology, Shahid Beheshti University G.C, Tehran, IR, Iran.

Received: 7 March 2020 Accepted: 15 June 2020

Published online: 22 June 2020

\section{References}

1. Huarte M, Guttman M, Feldser D, Garber M, Koziol MJ, Kenzelmann-Broz D, Khalil AM, Zuk O, Amit I, Rabani M, et al. A large intergenic noncoding RNA induced by p53 mediates global gene repression in the p53 response. Cell. 2010;142(3):409-19.

2. Chillón I, Pyle AM. Inverted repeat Alu elements in the human lincRNAp21 adopt a conserved secondary structure that regulates RNA function. Nucleic Acids Res. 2016;44(19):9462-71.

3. Wu G, Cai J, Han Y, Chen J, Huang ZP, Chen C, Cai Y, Huang H, Yang Y, Liu $Y$, et al. LincRNA-p21 regulates neointima formation, vascular smooth muscle cell proliferation, apoptosis, and atherosclerosis by enhancing p53 activity. Circulation. 2014;130(17):1452-65.

4. Dimitrova N, Zamudio JR, Jong RM, Soukup D, Resnick R, Sarma K, Ward AJ, Raj A, Lee JT, Sharp PA, et al. LincRNA-p21 activates p21 in cis to promote Polycomb target gene expression and to enforce the G1/S checkpoint. Mol Cell. 2014;54(5):777-90.

5. Yoon JH, Srikantan S, Gorospe M. MS2-TRAP (MS2-tagged RNA affinity purification): tagging RNA to identify associated miRNAs. Methods. 2012;58(2):81-7.

6. Jia M, Jiang L, Wang YD, Huang JZ, Yu M, Xue HZ. lincRNA-p21 inhibits invasion and metastasis of hepatocellular carcinoma through Notch signaling-induced epithelial-mesenchymal transition. Hepatol Res. 2016;46(11):1137-44

7. Wang $X$, Ruan $Y$, Wang $X$, Zhao W, Jiang $Q$, Jiang $C$, Zhao $Y, X u$, , Sun F, Zhu $Y$, et al. Long intragenic non-coding RNA lincRNA-p21 suppresses development of human prostate cancer. Cell Prolif. 2017;50(2):e12318.

8. Ozgur E, Mert U, Isin M, Okutan M, Dalay N, Gezer U. Differential expression of long non-coding RNAs during genotoxic stress-induced apoptosis in HeLa and MCF-7 cells. Clin Exp Med. 2013;13(2):119-26.

9. Jin S, Yang $X$, Li J, Yang W, Ma H, Zhang Z. p53-targeted lincRNA-p21 acts as a tumor suppressor by inhibiting JAK2/STAT3 signaling pathways in head and neck squamous cell carcinoma. Mol Cancer. 2019;18(1):38.

10. Hall JR, Messenger ZJ, Tam HW, Phillips SL, Recio L, Smart RC. Long noncoding RNA lincRNA-p21 is the major mediator of UVB-induced 
and p53-dependent apoptosis in keratinocytes. Cell Death Dis. 2015;6(3):e1700-e1700.

11. Tang S-S, Zheng B-Y, Xiong X-D. LincRNA-p21: implications in human diseases. Int J Mol Sci. 2015;16(8):18732-40.

12. Chen $Y$, Wei $G$, Xia H, Yu H, Tang Q, Bi F. Down regulation of lincRNA-p21 contributes to gastric cancer development through Hippo-independent activation of YAP. Oncotarget. 2017;8(38):63813-24

13. Blume CJ, Hotz-Wagenblatt A, Hullein J, Sellner L, Jethwa A, Stolz T, Slabicki M, Lee K, Sharathchandra A, Benner A, et al. p53-dependent non-coding RNA networks in chronic lymphocytic leukemia. Leukemia. 2015;29(10):2015-23.

14. Castellano JJ, Navarro A, Vinolas N, Marrades RM, Moises J, Cordeiro A, Saco A, Munoz C, Fuster D, Molins L, et al. LincRNA-p21 impacts prognosis in resected non-small cell lung cancer patients through angiogenesis regulation. J Thorac Oncol. 2016;11(12):2173-82.

15. Zhai H, Fesler A, Schee K, Fodstad O, Flatmark K, Ju J. Clinical significance of long intergenic noncoding RNA-p21 in colorectal cancer. Clin Colorectal Cancer. 2013;12(4):261-6.

16. Peng W, Wu J, Feng J. LincRNA-p21 predicts favorable clinical outcome and impairs tumorigenesis in diffuse large B cell lymphoma patients treated with R-CHOP chemotherapy. Clin Exp Med. 2017;17(1):1-8.

17. Helsmoortel H, Everaert C, Lumen N, Ost P, Vandesompele J. Detecting long non-coding RNA biomarkers in prostate cancer liquid biopsies: hype or hope? Non-coding RNA Res. 2018;3(2):64-74.

18. Tran UM, Rajarajacholan U, Soh J, Kim T, Thalappilly S, Sensen CW, Riabowol K. LincRNA-p21 acts as a mediator of ING1b-induced apoptosis. Cell Death Dis. 2015;6(3):e1668-e1668.

19. Yang T, Zhang W, Wang L, Xiao C, Guo B, Gong Y, Liang X, Huang D, Li Q, Nan Y, et al. Long intergenic noncoding RNA-p21 inhibits apoptosis by decreasing PUMA expression in non-small cell lung cancer. J Int Med Res. 2019;47(1):481-93.

20. Ma J, Feng Y, Liu Y, Li X. PUMA and survivin are involved in the apoptosis of HepG2 cells induced by microcystin-LR via mitochondria-mediated pathway. Chemosphere. 2016;157:241-9.

21. Vavrova J, Rezacova M. Importance of proapoptotic protein PUMA in cell radioresistance. Folia Biol. 2014;60(2):53-6.

22. Ye Y, Peng Y, Li Y, Liu C, Xu Y, Li W. Effect of lincRNA-p21 targeting HIF-1a on biological functions of liver cancer cells. Oncol Lett. 2019;17(6):4964-8.

23. Zhang Y, Miao Y, Shang M, Liu M, Liu R, Pan E, Pu Y, Yin L. LincRNA-p21 leads to $\mathrm{G} 1$ arrest by p53 pathway in esophageal squamous cell carcinoma. Cancer Manag Res. 2019;11:6201-14.

24. Xia W, Zhuang L, Deng X, Hou M. Long noncoding RNAp21 modulates cellular senescence via the Wnt/betacatenin signaling pathway in mesenchymal stem cells. Mol Med Rep. 2017;16(5):7039-47.

25. Yoon JH, Abdelmohsen $\mathrm{K}$, Srikantan S, Yang X, Martindale JL, De S, Huarte M, Zhan M, Becker KG, Gorospe M. LincRNA-p21 suppresses target mRNA translation. Mol Cell. 2012;47(4):648-55.

26. Yang F, Zhang H, Mei Y, Wu M. Reciprocal regulation of HIF-1alpha and lincRNA-p21 modulates the Warburg effect. Mol Cell. 2014;53(1):88-100.

27. Wang J, Lei Z, Guo Y, Wang T, Qin Z, Xiao H, Fan L, Chen D, Bian X, Liu J, et al. MiRNA-regulated delivery of lincRNA-p21 suppresses $\beta$-catenin signaling and tumorigenicity of colorectal cancer stem cells. Oncotarget. 2015:6(35):37852-70.

28. Bao X, Wu H, Zhu X, Guo X, Hutchins AP, Luo Z, Song H, Chen Y, Lai K, Yin $M$, et al. The p53-induced lincRNA-p21 derails somatic cell reprogramming by sustaining $\mathrm{H} 3 \mathrm{~K} 9 \mathrm{me} 3$ and $\mathrm{CpG}$ methylation at pluripotency gene promoters. Cell Res. 2015;25(1):80-92.

29. Isin M, Ozgur E, Cetin G, Erten N, Aktan M, Gezer U, Dalay N. Investigation of circulating IncRNAs in B-cell neoplasms. Clin Chim Acta. 2014:431:255-9.

30. Fang L, Wu S, Zhu X, Cai J, Wu J, He Z, Liu L, Zeng M, Song E, Li J, et al. MYEOV functions as an amplified competing endogenous RNA in promoting metastasis by activating TGF-beta pathway in NSCLC. Oncogene. 2019;38(6):896-912

31. Wei L, Wu T, He P, Zhang JL, Wu W. LncRNA ATB promotes the proliferation and metastasis of lung cancer via activation of the p38 signaling pathway. Oncol Lett. 2018;16(3):3907-12.

32. Liao Y, Cheng S, Xiang J, Luo C. InCRNA CCHE1 increased proliferation, metastasis and invasion of non-small lung cancer cells and predicted poor survival in non-small lung cancer patients. Eur Rev Med Pharmacol Sci. 2018;22(6):1686-92.

33. Ding G, Peng Z, Shang J, Kang Y, Ning H, Mao C. LincRNA-p21 inhibits invasion and metastasis of hepatocellular carcinoma through miR-9/Ecadherin cascade signaling pathway molecular mechanism. Onco Targets Ther. 2017;10:3241-7.

34. Nishida N, Yano H, Nishida T, Kamura T, Kojiro M. Angiogenesis in cancer. Vasc Health Risk Manag. 2006;2(3):213-9.

35. Rajabi M, Mousa SA. The role of angiogenesis in cancer treatment. Biomedicines. 2017;5(2):34

36. Kumar MM, Goyal R. LncRNA as a therapeutic target for angiogenesis. Curr Top Med Chem. 2017;17(15):1750-7.

37. Cui D, Zhu D, Ren H, Lin J, Yang M. LincRNA-p21 inhibits inflammatory response and angiogenesis through the NF-KB signaling pathway in rats with rheumatoid arthritis. Int J Clin Exp Med. 2018;11(2):1217-25.

38. Yu F, Guo Y, Chen B, Shi L, Dong P, Zhou M, Zheng J. LincRNA-p21 inhibits the Wnt/beta-catenin pathway in activated hepatic stellate cells via sponging MicroRNA-17-5p. Cell Physiol Biochem. 2017;41(5):1970-80.

39. Wang $X, X u Y$, Wang $X$, Jiang $C$, Han S, Dong $K$, Shen M, Xu D. LincRNAp21 suppresses development of human prostate cancer through inhibition of PKM2. Cell Prolif. 2017;50(6):e12395.

40. Taylor BS, Schultz N, Hieronymus H, Gopalan A, Xiao Y, Carver BS, Arora VK, Kaushik P, Cerami E, Reva B, et al. Integrative genomic profiling of human prostate cancer. Cancer Cell. 2010;18(1):11-22.

41. Wang G, Li Z, Zhao Q, Zhu Y, Zhao C, Li X, Ma Z, Li X, Zhang Y. LincRNAp21 enhances the sensitivity of radiotherapy for human colorectal cancer by targeting the Wnt/beta-catenin signaling pathway. Oncol Rep. 2014;31(4):1839-45.

42. Yu F, Lu Z, Chen B, Dong P, Zheng J. Identification of a novel lincRNAp21-miR-181b-PTEN signaling cascade in liver fibrosis. Mediat Inflamm. 2016:2016:9856538.

43. Ao $X$, Jiang $M$, Zhou J, Liang $H$, Xia H, Chen G. lincRNAp21 inhibits the progression of nonsmall cell lung cancer via targeting miR175p. Oncol Rep. 2019;41(2):789-800.

44. Wang KC, Chang HY. Molecular mechanisms of long noncoding RNAs. Mol Cell. 2011;43(6):904-14.

45. Salmena L, Poliseno L, Tay Y, Kats L, Pandolfi PP. A ceRNA hypothesis: the Rosetta Stone of a hidden RNA language? Cell. 2011;146(3):353-8.

46. Yamamura S, Imai-Sumida M, Tanaka Y, Dahiya R. Interaction and crosstalk between non-coding RNAs. Cell Mol Life Sci. 2018;75(3):467-84.

47. Ye Y, He X, Lu F, Mao H, Zhu Z, Yao L, Luo W, Sun X, Wang B, Qian C, et al. A lincRNA-p21/miR-181 family feedback loop regulates microglial activation during systemic LPS- and MPTP-induced neuroinflammation. Cell Death Dis. 2018;9(8):803-803.

48. Xu X, Zhuang C, Wu Z, Qiu H, Feng H, Wu J. LincRNA-p21 inhibits cell viability and promotes cell apoptosis in Parkinson's disease through activating a-synuclein expression. Biomed Res Int. 2018;2018:8181374-8181374.

49. Chou SD, Murshid A, Eguchi T, Gong J, Calderwood SK. HSF1 regulation of beta-catenin in mammary cancer cells through control of HuR/elavL1 expression. Oncogene. 2015;34(17):2178-88.

50. Deng K, Wang H, Guo X, Xia J. The cross talk between long, non-coding RNAs and microRNAs in gastric cancer. Acta Biochim Biophys Sin. 2015:48(2):111-6.

51. Tang L, Ding J, Zhou G, Liu Z. LncRNAp21 promotes chondrocyte apoptosis in osteoarthritis by acting as a sponge for miR451. Mol Med Rep. 2018;18(6):5295-301.

52. Zhang J, Cao X, Wang S, Aizimaiti M, Xielifu R, Liu J. LincRNA-p21 sponges miR-18b to promote the progression of diabetic nephropathy. Am J Transl Res. 2018;10:1481-9.

53. Cesana M, Cacchiarelli D, Legnini I, Santini T, Sthandier O, Chinappi M, Tramontano A, Bozzoni I. A long noncoding RNA controls muscle differentiation by functioning as a competing endogenous RNA. Cell. 2011;147(2):358-69.

\section{Publisher's Note}

Springer Nature remains neutral with regard to jurisdictional claims in published maps and institutional affiliations. 